\title{
Propagation of partially coherent radiation using Wigner functions
}

\author{
Boaz Nash, ${ }^{*}$ Nicholas Goldring, Jonathan Edelen $\odot$, and Stephen Webb \\ RadiaSoft LLC, 3380 Mitchell Lane, Boulder, Colorado 80301, USA \\ Rafael Celestre \\ ESRF-The European Synchrotron, 71 Avenue des Martyrs, 38000 Grenoble, France
}

(Received 7 September 2020; accepted 23 December 2020; published 19 January 2021)

\begin{abstract}
Undulator radiation from synchrotron light sources must be transported down a beam line from the source to the sample. A partially coherent photon beam may be represented in phase space using a Wigner function, and its transport may use some similar techniques that are familiar in particle beam transport. We describe this process in the case that the beam line is composed of linear focusing and defocusing sections as well as apertures. We present a compact representation of the beam line map involving linear transformations and convolutions. We create a 1:1 imaging system with a single slit on the image plane and observe the radiation downstream to it. We propagate a Gaussian beam and undulator radiation down this sample beam line, drawing parameters from current and future ultra low emittance light sources. We derive an analytic expression for the partially coherent Gaussian case including passage through a single slit aperture. We benchmark the Wigner function calculation against the analytical expression and a partially coherent calculation in the synchrotron radiation workshop (SRW) code.
\end{abstract}

DOI: 10.1103/PhysRevAccelBeams.24.010702

\section{INTRODUCTION}

Synchrotron radiation sources, either storage ring or FEL-based, require optical beam lines to transport the radiation to the experimental sample. As performance of these sources is being pushed to lower emittance and higher coherence, new attention is being drawn to the beam line performance and optics modeling [1].

Models of radiation transport through the beam line elements exist in a hierarchy of levels of accuracy and complexity. At the simplest level, one can use analytical formulas to propagate beam sizes, divergences and coherence lengths through an idealized beam line (see [2]). At the next level is the geometric optics description using a ray-tracing approach (e.g. SHADOW [3]). At a higher level of complexity, one may use a physical optics approach which requires wavefront propagation software (e.g. SRW [4]). The wavefront propagation allows the inclusion of diffraction effects in coherent optics, but is more computationally intensive than the ray-tracing approach.

To go beyond optics of coherent wavefronts, accurate representation of radiation requires the model to include partial coherence which may be treated using considerations

\footnotetext{
*bnash@radiasoft.net

Published by the American Physical Society under the terms of the Creative Commons Attribution 4.0 International license. Further distribution of this work must maintain attribution to the author(s) and the published article's title, journal citation, and DOI.
}

of statistical optics [5,6]. For synchrotron radiation, this partial coherence results from the finite electron beam size causing randomness in the phase of the emitted radiation. In the wavefront propagation method, this may be taken into account by propagating multiple initial wavefronts, either via a sampling of the phase space of initial electron beam [7] or by a coherent mode decomposition [8-10].

Another approach to treating partially coherent radiation involves the use of Wigner functions. The Wigner function formalism for synchrotron radiation was pioneered by K.-J. Kim [11]. The Wigner function was originally developed in quantum mechanics [12-14], as an alternative phase-space representation to the density operator. In an optical context, the Wigner function may represent the types of systems described in statistical optics, where multiple wavefronts are simultaneously present with random phase relations between them. The properties of Wigner functions and the relation between the quantum mechanics and optics contexts are described by Bazarov [15].

Although Wigner functions for fully and partially coherent synchrotron radiation have been computed, they have not been widely used in the propagation down beam lines. In this paper, we demonstrate propagation of the fully and partially coherent Wigner functions through a simplified beam line. We compute beam line maps that take radiation at the source to radiation at the sample. We limit ourselves to linear maps, (so-called ABCD matrix, in the optics literature $[16,17]$ ) under which the Wigner function transforms in a straightforward manner. We will also consider 
physical apertures, focusing on the case of single slits, an important element in most x-ray beam lines. We assume separable radiation (no coupling between horizontal and vertical propagation) such that we may work with $2 \mathrm{D}$ Wigner functions $[15,18]$. The extension to higher dimensionality is straightforward. Including nonlinear elements, such as spherical aberrations in a lens, will be a topic of future work.

As the size of the electron beam increases, the Wigner function becomes dominated by the Gaussian electron beam and the coherence decreases. To understand this transition, we consider Gaussian initial Wigner functions. Gaussian Wigner functions have been treated in detail already in the literature and go by the name of "Gauss Schell models" [19]. After passing through an aperture, however, they are no longer Gaussian. We are able to derive an analytic expression for the diffraction of a partially coherent Gaussian through a single slit and the expected intensity distribution on a subsequent screen. We may thus validate our algorithms for linear transport and passage through an aperture. In addition, we may compare the analytical Gaussian result to the case of undulator radiation and gain understanding as to when the Gaussian result may be adequate in simulation. Finally, we validate our undulator radiation transport using our simplified Wigner function map method to a partially coherent SRW calculation.

\section{X-RAY BEAM LINE MODELING}

A synchrotron radiation beam line model represents the optical system that transports X-rays from the electron beam source all the way to the sample, passing through a series of optical elements along the way. There are several methods of approaching this modeling task: we survey physical optics in the Appendix A, for example. We start with a summary of the properties of the radiation Wigner function. Next, we provide a brief discussion of linear geometric optics including apertures. Finally, we show how to evolve the Wigner function under the action of a matrix-aperture beam line.

Consider an optical ray and attribute to it a wavelength $\lambda$ following a trajectory starting at position $s=0$ where the radiation is created and ending at $S=L$ at the end of the beam line. This trajectory will in general not be straight due to reflections from mirrors and gratings and passing through other optical elements. At each position $s$, along the trajectory, we assign transverse phase space coordinates, $\vec{z}$,

$$
\vec{z}=\left(\begin{array}{c}
x \\
\theta_{x} \\
y \\
\theta_{y}
\end{array}\right),
$$

where $x$ and $y$ are transverse coordinates, $\theta_{x}$ and $\theta_{y}$ are corresponding angles with $\theta_{x}=\frac{d x}{d s}$ and $\theta_{y}=\frac{d y}{d s}$.

\section{A. Radiation Wigner functions}

We represent the radiation along the trajectory by means of a Wigner function $W(\vec{z})$. Many of the properties of Wigner functions have been reviewed by Bazarov [15]. We mention several of them so that our treatment here is selfcontained. First, the Wigner function is normalized:

$$
\int d \vec{z} W(\vec{z})=1
$$

We adopt this normalization for clarity of presentation and close connection to the corresponding quantum mechanical formalism. The Wigner function is related to the brightness (or brilliance) function $\mathcal{B}$ by means of an overall factor of the radiation flux $\phi$ :

$$
\mathcal{B}(\vec{z} ; s)=\phi(s) W(\vec{z} ; s) .
$$

Thus, as the radiation moves along the beam line, progressing in $s$ and passes through absorbing elements (such as apertures that we consider here), the flux $\phi$ will reduce, but the normalization of $W(\vec{z} ; s)$ will remain constant.

Now, suppose we know the Wigner function at $s=0$, $W_{0}\left(x, \theta_{x}, y, \theta_{y}\right)$. We will assume that $W_{0}$ is separable; that is that $W_{0}$ obeys $^{2}$

$$
W_{0}\left(x, \theta_{x}, y, \theta_{y}\right)=W_{x}\left(x, \theta_{x}\right) W_{y}\left(y, \theta_{y}\right) .
$$

In the examples we consider, the Wigner function remains separable throughout the beam line, and thus we may consider propagation of the components separately. We thus refer to $W_{x}\left(x, \theta_{x}\right)$ or $W_{y}\left(y, \theta_{y}\right)$ simply by $W(x, \theta)$.

Now, suppose that $W(x, \theta)$ represents fully coherent radiation. Then, there exists an electric field $E(x)$ such that

$$
W(x, \theta)=\frac{1}{\lambda} \int_{-\infty}^{\infty} E^{*}\left(x-\frac{\phi}{2}\right) E\left(x+\frac{\phi}{2}\right) e^{-\frac{2 \pi i}{\lambda} \phi \theta} d \phi .
$$

We may write this equation in an operator form:

$$
W(x, \theta)=\mathcal{W}[E(x)],
$$

where we refer to $\mathcal{W}$ as the Wigner transform operator, or $W(x, \theta)$ as the Wigner function associated with $E(x)$. In the

\footnotetext{
${ }^{1}$ Note that we will ignore the longitudinal phase space coordinates in this work. A 6D phase space treatment would include the longitudinal position and relative wavelength deviation. In particular we ignore pulse length effects and assume a monochromatic beam.

${ }^{2}$ Note that for the case of undulator radiation, this condition only approximately holds. On resonance, the condition is satisfied to within a few percent over a wide range of emittance values. See Fig. 6 in [18].
} 
case of fully coherent radiation, the electric field may be reconstructed from the Wigner function as follows [15]

$$
E^{*}(x) E(0)=\frac{1}{\lambda} \int_{-\infty}^{\infty} W\left(\frac{x}{2}, \theta\right) e^{\frac{2 \pi i x}{\lambda} x} d \theta .
$$

Now, in the case where $W$ is partially coherent, there does not exist a single, well-defined wavefront associated with the Wigner function. Rather, there exists a whole sequence of fields $E_{j}(x)$ with $j=1 \ldots \infty$. The Wigner function is given as the (infinite) sum of the Wigner transforms associated with the $E_{j}$ :

$$
W(x, \theta)=\sum_{j} \mathcal{W}\left[E_{j}(x)\right]
$$

The decomposition of a given partially coherent Wigner function into a set of underlying fields $E_{j}$ is not generally unique. In the case that the fields are orthogonal, and are eigenvectors of the underlying transport operator, the fields are referred to as modes (see Sec. 4.7 in [20]). The process of finding an astute choice of such modes is known as "coherent mode decomposition." In constructing the partially coherent Wigner function for synchrotron radiation, one must integrate over the electron beam distribution [see later Eqs. (42)-(44)]. Thus, although these wavefronts are not orthogonal, we may also consider the decomposition into single electron modes as a form of coherent mode decomposition satisfying Eq. (8).

From the Wigner function, one may compute the quantity, $\mu$, known as the degree of coherence via the following expression:

$$
\mu^{2}=\lambda \int W^{2}(x, \theta) d x d \theta
$$

In the fully coherent case where $W$ may be derived from an electric field, we find $\mu=1$. For the partially coherent case, $\mu<1$.

\section{B. Linear geometric optics}

Along an x-ray beam line, there are optical elements which the radiation will interact with. We include these in our model by including a varying optical path difference, that is, a phase and amplitude modulation, as a function of the Cartesian coordinates $(x, y ; s)$. This optical path length difference is often a function of the index of refraction $n(x, y ; s)$ for transmission elements. In addition we will include physical apertures, which allow radiation within a certain transverse region to pass unimpeded, and absorb all radiation outside of that region. The aperture elements may be described by transfer functions $t(x, y)$ which describe the region where rays may pass, and where they are absorbed. In fact, we may allow more general aperture elements with values between 0 and 1 in which the intensity of the ray may be reduced but not fully absorbed.

Now consider another ray, starting at a different initial condition $\vec{Z}_{0}$. The evolution of this ray down the beam line may be described by the action of the following Hamiltonian $^{3}$

$$
H\left(x, \theta_{x}, y, \theta_{y} ; s\right)=-\sqrt{n^{2}(x, y ; s)-\theta_{x}^{2}-\theta_{y}^{2}} .
$$

with $\theta_{x}$ and $\theta_{y}$ playing the role of momenta, and with position along the trajectory $s$ as independent variable. $n(x, y ; s)$ is the local index of refraction that the radiation is passing through. The result of this Hamiltonian formulation for geometric optics is that the offset ray will follow Hamilton's equations:

$$
\dot{Z}_{i}=J_{i j} \frac{\partial H}{\partial Z_{j}}
$$

with summation over the repeated index $j$ implied and the dot representing $\frac{d}{d s}$. For 4D phase space (in the case that we ignore variation in the $z$ and $\delta$ coordinates), the matrix $J$ is given by

$$
J=\left(\begin{array}{cccc}
0 & 1 & 0 & 0 \\
-1 & 0 & 0 & 0 \\
0 & 0 & 0 & 1 \\
0 & 0 & -1 & 0
\end{array}\right)
$$

If we allow arbitrary index of refraction $n(x, y)$ in our model beam line, then the equations of motion will be nonlinear. For the purposes of this paper, we will restrict to the approximation that the index of refraction varies quadratically, leading to linear equations of motion for the ray tracing. In particular, as our model for the index of refraction $n(x, y ; s)$, we will assume it to be constant along the optical axis and then to fall off quadratically in the transverse directions. Thus, we parametrize it as follows ${ }^{4}$ :

$$
n(x, y ; s)=n_{0}(s)-\kappa_{x}(s) x^{2}-\kappa_{y}(s) y^{2} .
$$

Expanding the Hamiltonian for small angles, to quadratic order, we find

\footnotetext{
${ }^{3}$ This Hamiltonian may be derived from the formulas for scalar diffraction theory under the paraxial approximation. One published derivation starting from a Lagrangian, and converting to a Hamiltonian is given in Ref. [21] by A. Dragt. A second derivation by A. Dragt in which an "operator square root" is taken is available in unpublished notes [22].

${ }^{4}$ Note that we leave out a coupling term in the Hamiltonian proportional to $x y$ so that the separable condition is satisfied.
} 
$H\left(x, y, \theta_{x}, \theta_{y} ; s\right) \approx-n_{0}(s)+\frac{\theta_{x}^{2}+\theta_{y}^{2}}{2 n_{0}}+\kappa_{x}(s) x^{2}+\kappa_{y}(s) y^{2}$.

As mentioned, this Hamiltonian will lead to linear equations of motion. The solution may thus be expressed in matrix form as

$$
\vec{Z}(s)=M(s) \vec{Z}_{0}
$$

One may solve these equations and produce a linear map for a given beam line section. All the optical elements (excluding the apertures which we deal with separately are thus captured in the transfer matrix $M(s)$ varying along the beam line. Because the ray tracing dynamics are derived from a Hamiltonian, we are assured that the resulting transfer matrix is symplectic. That is:

$$
M(s)^{T} J M(s)=J,
$$

for all $s$ along the beam line. The matrix $J$ is given in Eq. (12).

Although we have formulated this section in terms of a Hamiltonian theory to bring out some of the formal properties of the propagation, the transfer matrix may also be computed for realistic beam lines using ray tracing software. See [23] for an example of this calculation for a KB mirror system.

In the next section, we describe the evolution of the Wigner function under the matrix $M$. This covers both the fully coherent and partially coherent case. In the fully coherent case, a different formalism is possible for the propagation: that of the linear canonical transform (LCT). We outline this in Appendix A.

\section{Partially coherent propagation with Wigner function}

The evolution equation for the Wigner function is given as follows [13]

$$
\frac{\partial W\left(x, \theta_{x}, y, \theta_{y} ; s\right)}{\partial s}=[W, H]_{\star},
$$

where the Moyal bracket is defined for arbitrary phase space functions $f$ and $g$ as

$$
[f, g]_{\star}=\frac{1}{i \Uparrow}(f \star g-g \star f),
$$

and the Moyal star is given by

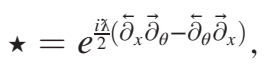

with the arrows representing action of the derivative, either to the left or right, depending on arrow orientation.
Fortunately, in the case of a quadratic Hamiltonian, evolution of the Wigner function is much simpler and more intuitive. The Moyal bracket reduces to the Poisson bracket giving classical evolution (again using the quantum/ classical mechanics analogy). One finds that the motion in phase space is a linear transformation. These considerations allow us to formulate our approach. In particular, consider a beam line where the geometric optics is defined by a transfer matrix $M$ acting on the phase space vector $\vec{z}$ :

$$
\vec{z}_{f}=M \vec{z}_{i}
$$

The Wigner function evolves along this beam line according to

$$
W_{f}(\vec{z})=W_{i}(M \vec{z}) .
$$

We may describe this transformation with the operator, $U_{M}$, defined as

$$
U_{M}(W(\vec{z}))=W(M \vec{z}) .
$$

By performing a change of variables, one may show that the degree of coherence $\mu$ is conserved under linear transport $U_{M}$. That is,

$$
\mu(W(\vec{z}))=\mu(W(M \vec{z})) .
$$

The degree of coherence is not conserved after passing through an aperture, which we now describe.

We would now like to consider the way in which Wigner functions are impacted by physical apertures. As described by Bazarov, for the electric field, the effect of the aperture is given by

$$
E_{s^{\prime}}(\vec{x})=E_{s}(\vec{x}) t(\vec{x})
$$

where $t(\vec{x})$ is the transmission function of the aperture.

In terms of the Wigner function, the action of the aperture is given by the partial convolution (in the angular variable) of the aperture Wigner function:

$$
W_{s^{\prime}}=W_{s^{*} \theta} W_{t} \equiv \mathcal{A}_{t} W_{s} .
$$

The aperture Wigner function $W_{t}$ is given by applying the Wigner transform to the aperture transmission function. That is, we apply Eq. (5) where the aperture transmission function $t(x)$ plays the role of the electric field.

Since an aperture results in absorption of radiation, the normalization of the Wigner function would change after passing through. As given in Eq. (3), the Wigner function is related to the brightness by a factor of the total flux. For simplicity, we will ignore the changing value of flux along the beam line and consider Wigner function to be consistently normalized throughout. Thus we normalize the 


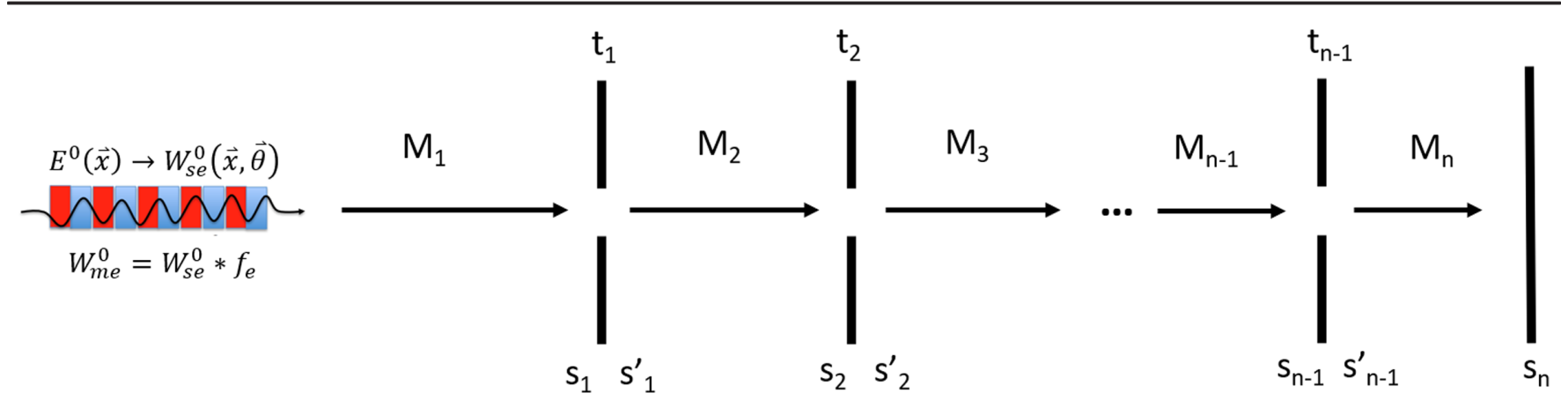

FIG. 1. Matrix aperture beam line schematic.

transmission function according to Eq. (B2) and thus $\mathcal{W}\left(t_{j}\right)$ will be normalized according to Eq. (B7). Because of the different normalization, the Wigner transform of a transmission function could be considered as a sort of filter that acts on the incoming radiation Wigner distribution function (WDF). Thus, part of the work of propagation of WDFs through beam lines including apertures involves the calculation of the Wigner filter functions. We will give an example of this function for the single slit aperture in a later section. A substantial part of the understanding about diffraction effects can be gleaned by examination of these Wigner filter functions.

\section{Matrix-aperture beam lines}

Consider the beam line schematic as shown in Fig. 1 consisting of an undulator source with an electron beam and subsequent sections which may be described by matrices, $M_{j}$, and apertures with transmission function, $t_{j}(x)$. An electron beam with distribution $f_{e}(\vec{z})$ passes through an undulator producing synchrotron radiation. Let $E^{0}(\vec{x})$ be the electric field produced by a single electron as it appears at the center of the undulator. We may now construct the multi-electron Wigner function for the undulator radiation as will be described in Sec. IVA [see Eq. (44)].

We consider an axial ray coming from the center of the undulator and proceeding along the beam line until the final observation plane located at position $s_{n}$. We note that the optical axis described by this axial ray is not necessarily a straight line. In particular, mirrors will cause angular deviations from the central trajectory and at each point, the transverse coordinates are relative to the direction of the central ray. Along the beam line, there are apertures located at positions $s_{1}$ through $s_{n-1}$ which are represented by transmission functions $t_{1}$ through $t_{n-1}$. For the purpose of our simplified beam line, nonlinear aberrations will be ignored and we will assume that the transport between apertures $j-1$ and $j$ may be represented by the matrix $M_{j}$.

Following Eq. (22), we find an operator for this beam line section given by $U_{M_{j}}$ that simply transports the phase space by applying the matrix $M_{j}$. We have thus defined the operator for propagation through sections of linear transport, $U_{M_{j}}$, that may include mirrors, lenses, and other elements when remaining close to the optical axis. Likewise, the apertures may also be represented by operators, $\mathcal{A}_{t_{j}}$, as given by Eq. (25). The operator for the entire simplified beam line may then be given by

$$
\mathcal{O}_{B L}=U_{M_{n}} \mathcal{A}_{t_{n-1}} U_{M_{n-1}} \ldots \mathcal{A}_{t_{2}} U_{M_{2}} \mathcal{A}_{t_{1}} U_{M_{1}} \text {. }
$$

Then the fully coherent and partially coherent simulations can be simply written as

$$
\begin{gathered}
W_{s e, n}=\mathcal{O}_{B L} W_{s e}^{0} \\
W_{m e, n}=\mathcal{O}_{B L} W_{m e}^{0} .
\end{gathered}
$$

\section{EXAMPLES-GAUSSIAN RADIATION BEAM}

We consider Gaussian radiation (GR) and undulator radiation (UR) propagating through a simple matrix-aperture beam line doing a 1:1 imaging of the source with a horizontal slit at the image plane and observing the radiation downstream to it. A schematic for this beam line is depicted in Fig. 2 and the corresponding source and beam line parameters are provided in Table I.

We first illustrate many of the useful properties of radiation Wigner function distribution (WFD) propagation with the example of Gaussian radiation. The one-to-one imaging section preserves the coherence properties ${ }^{5}$ and thus the first element to consider is the single slit aperture. Propagation through the aperture and subsequent propagation through free space allows us to observe the impact of decreasing coherence on the WFD and corresponding intensity pattern. As the divergence becomes sufficiently large, the oscillations in $\theta$ in the aperture Wigner filter are washed out, and coherent diffraction effects are seen to be destroyed. The GR case has the advantage of exploiting the analytical expressions previously derived for benchmarking our numerical WFD transport methods.

\footnotetext{
${ }^{5}$ This assumes that there is no beam cropping and that the imaging system can resolve the radiation source.
} 


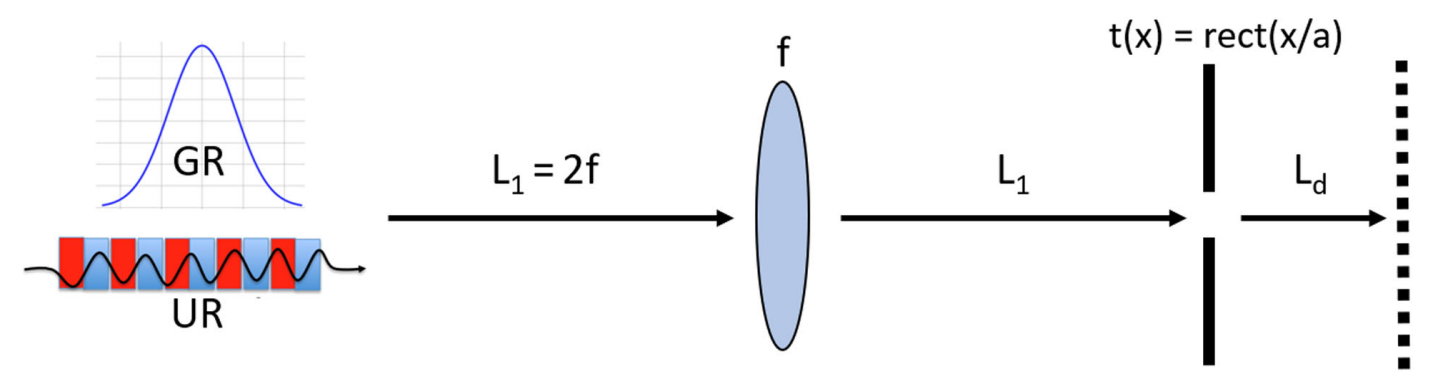

FIG. 2. Simple matrix-aperture beam line example.

We then explore the more complex case of UR propagating through this beam line. Changing the electron beam emittance allows us to control the degree of partial coherence. At small electron beam emittances, the WFD is dominated by the single electron WFD, but as the electron beam emittance increases, this structure becomes less relevant and the WFD asymptotically reduces to the GR case. In order to confirm the accuracy of our calculations, we have set up the same beam line model in SRW. Due to the separability of the horizontal and vertical components, we are able to demonstrate a direct comparison between SRW and our own partially coherent WFD based propagation results. Our Wigner function based methods are done in two phase space dimensions and rely on the separability of the radiation to be able to compare to SRW. A more detailed analysis of the computational complexity of the different methods is discussed in Sec. V.

We first consider a Gaussian beam propagating through the simple matrix aperture beam line where we can compare our numerical results to the analytical expression given by Eq. (40).

TABLE I. Numerical simulation parameters.

\begin{tabular}{lcc}
\hline \hline Undulator radiation & & \\
\hline Length, $L_{u}$ & 2.31 & $\mathrm{~m}$ \\
Period, $\lambda_{u}$ & 0.033 & $\mathrm{~m}$ \\
Max. field, $B$ & 0.7 & $\mathrm{~T}$ \\
Deflection parameter, $k$ & 2.157 & \\
First resonant energy, $E_{1}$ & 3.115 & $\mathrm{keV}$ \\
Wavelength, $\lambda_{1}$ & 3.98 & $\AA$ \\
Electron beam $($ APS- $U$ ) & & \\
Energy, $E$ & 6.0 & $\mathrm{GeV}$ \\
Current, $I$ & 200 & $\mathrm{~mA}$ \\
Horizontal emittance, $\epsilon_{x}$ & 42.2 & $\mathrm{pm}$ \\
Horizontal RMS size, $\sigma_{x}$ & 14.44 & $\mu \mathrm{m}$ \\
Horizontal RMS divergence, $\sigma_{x^{\prime}}$ & 2.92 & $\mu \mathrm{rad}$ \\
Vertical emittance, $\epsilon_{y}$ & 4.20 & $\mathrm{pm}$ \\
Vertical RMS size, $\sigma_{y}$ & 2.82 & $\mu \mathrm{m}$ \\
Vertical RMS divergence, $\sigma_{y^{\prime}}$ & 1.49 & $\mu \mathrm{rad}$ \\
Beam line parameters & & \\
Distance to lens, $L_{1}$ & 30.0 & $\mathrm{~m}$ \\
Lens focal length, $f$ & 15.0 & $\mathrm{~m}$ \\
Aperture width, a & 16.7 & $\mu \mathrm{m}$ \\
Final drift length, $L_{d}$ & 0.1 & $\mathrm{~m}$ \\
\hline \hline
\end{tabular}

\section{A. Analytic calculation for Gaussian radiation with single slit aperture}

In this section, we derive an analytic expression for the propagation of a Gaussian Wigner function through a single slit aperture of width $a$. We are able to find an analytic expression for the radiation immediately after the slit as well as the radiation after having drifted some distance beyond. The aperture is described by the transmission function

$$
t(x)=\operatorname{rect}\left(\frac{x}{a}\right)
$$

with

$$
\operatorname{rect}(x)= \begin{cases}1, & |x|<\frac{1}{2} \\ \frac{1}{2} & |x|=\frac{1}{2} \\ 0, & |x|>\frac{1}{2}\end{cases}
$$

We derive an expression for the corresponding Wigner filter function in Appendix $\mathrm{D}^{6}$ :

$$
\begin{aligned}
W_{s s}(x, \theta) & =\operatorname{rect}\left(\frac{x}{a}\right) \frac{2 \sin \left[\frac{2 \pi \theta}{\lambda}(-2|x|+a)\right]}{\theta} \\
& \equiv \operatorname{rect}\left(\frac{x}{a}\right) \frac{2 \sin (\theta Q)}{\theta} \\
Q & =2 \pi \frac{a-2|x|}{\lambda} .
\end{aligned}
$$

Plots of the single slit aperture transmission function and corresponding WFD are displayed in Fig. 3.

Let us consider a partially coherent Gaussian Wigner function given by

$$
W(x, \theta)=\frac{1}{2 \pi \sigma_{x} \sigma_{\theta}} e^{-\frac{x^{2}}{2 \sigma_{x}^{2}}-\frac{\theta^{2}}{2 \sigma_{\theta}^{2}}} .
$$

Let us write the relationship between the position and angular spreads as

\footnotetext{
${ }^{6}$ This expression can also be found in Ref. [24].
} 

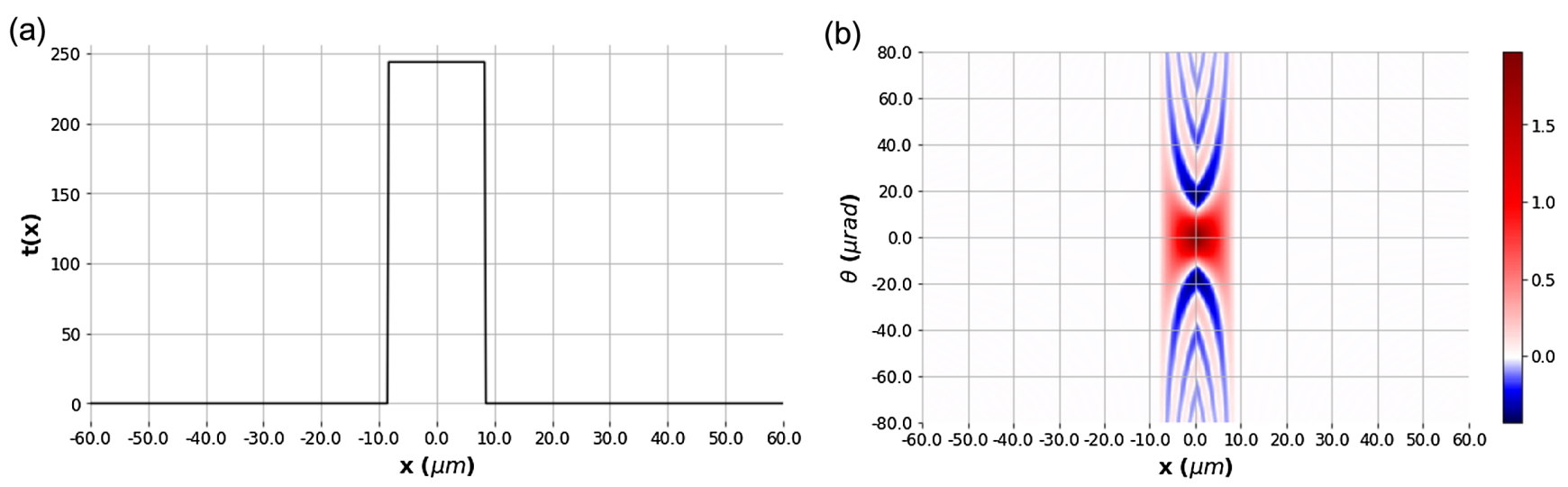

FIG. 3. Single slit aperture (a) transmission function and (b) corresponding Wigner filter function.

$$
\sigma_{x} \sigma_{\theta}=m^{2} \frac{\lambda}{4 \pi}
$$

where $m^{2}$ is the beam quality factor known in the laser optics literature. $^{7}$ In the case that $m^{2}=1$, the Wigner function represents a coherent wavefront. ${ }^{8}$ For $m^{2}>1$, this Wigner function represents partially coherent radiation.

To propagate the Wigner function through the slit, we perform a convolution of the Gaussian with Eq. (31) in the $\theta$ variable. That is,

$$
\begin{gathered}
W_{s_{1}^{\prime}}=W_{s_{1}{ }^{*} \theta} W_{s s} \\
=\operatorname{rect}\left(\frac{x}{a}\right) \frac{2}{2 \pi \sigma_{x} \sigma_{\theta}} e^{-\frac{x^{2}}{2 \sigma_{x}^{2}}} \mathcal{I}(\theta, Q),
\end{gathered}
$$

with

$$
\mathcal{I}(\theta, Q)=\int_{-\infty}^{\infty} e^{-\frac{(\theta-\tau)^{2}}{2 \sigma_{\theta}^{2}}} \frac{\sin (\tau Q)}{\tau} d \tau .
$$

This integral may be performed (see Appendix D for details) with the result

$$
\mathcal{I}(\theta, Q)=\pi e^{-\frac{\hat{\theta}^{2}}{2}} \operatorname{Im}\left\{i \operatorname{erf}\left(\frac{\hat{Q}+i \hat{\theta}}{\sqrt{2}}\right)\right\}
$$

where Im represents taking the imaginary part of the argument and $\hat{Q}=Q \sigma_{\theta}$ and $\hat{\theta}=\theta / \sigma_{\theta}$. Writing it all out explicitly to see the $x$ and $\theta$ dependence, and adding the drift following the aperture, we have:

\footnotetext{
${ }^{7}$ The beam quality factor is typically represented by a capital $M$. We have used a lower-case $m$ here so as not to confuse this quantity with the transfer matrix, $M$.

${ }^{8} \mathrm{We}$ note that for non-Gaussian wavefronts, in the coherent case, the product $\sigma_{x} \sigma_{\theta}$ exceeds $\frac{\lambda}{4 \pi}$. For undulator radiation, one has approximately $\frac{\lambda}{2 \pi}$. See e.g. [25-28].
}

$$
\begin{aligned}
W_{s_{1}^{\prime}}(x, \theta)= & \frac{1}{\sigma_{x} \sigma_{\theta}} \operatorname{rect}\left(\frac{x-L_{d} \theta}{a}\right) e^{-\frac{\left(x-L_{d} \theta\right)^{2}}{2 \sigma_{x}}-\frac{\theta^{2}}{2 \sigma_{\theta}^{2}}} \\
& \times \operatorname{Im}\left\{i \operatorname{erf}\left(\frac{\left(a-2\left|x-L_{d} \theta\right|\right) \frac{\sigma_{\theta}}{\lambda}+i \frac{\theta}{\sigma_{\theta}}}{\sqrt{2}}\right)\right\},
\end{aligned}
$$

where $L_{d}$ is the drift length following the single slit aperture.

From Eq. (9), and using the Gaussian Eq. (34), we find the expression for degree of coherence, $\mu$, before passing through the slit to be

$$
\mu=\frac{1}{m}
$$

Figure 4 shows the WFDs resulting from the convolution of GR with the aperture Wigner filter function displayed in Fig. 3(b). This calculation was performed on a discrete grid of size $n_{x}=4000$ by $n_{\theta}=4000$. Because the analytic expressions are available in the GR case, we are free to increase the resolution to a very fine level without incurring excessively large runtimes and memory demand. These results demonstrate the diminishing coherence effects for a fixed beam size and increasing divergences corresponding to increasing $m$ values.

Figure 5 shows the intuitive strength of the WFD method by illustrating the mechanism of diffraction in which the oscillations in $\theta$ give rise to interference effects following the drift. Before the drift, the oscillations of the Wigner functions in $\theta$ will cancel when performing a projection, but following the drift, they result in a large dip at the center of the intensity distribution which can be seen in Fig. 5(b). In Fig. 5(b), results are shown for increasing values of $\mathrm{m}^{2}$, showing how this interference effect disappears with decreasing coherence. In order to demonstrate the validity of our numerical computations, Figure 6 compares the spatial projections of the drifted GR WFD at $m^{2}=3$ calculated numerically and analytically. Because the 

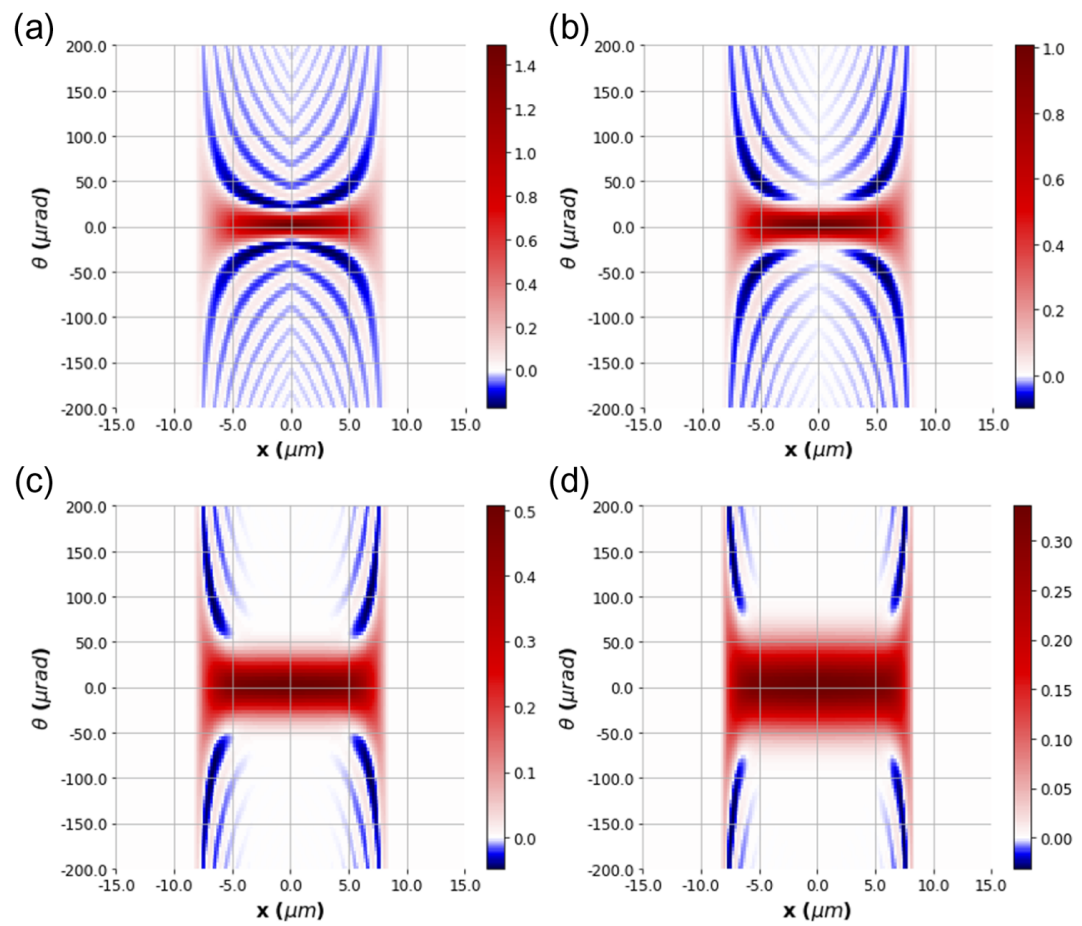

(d)
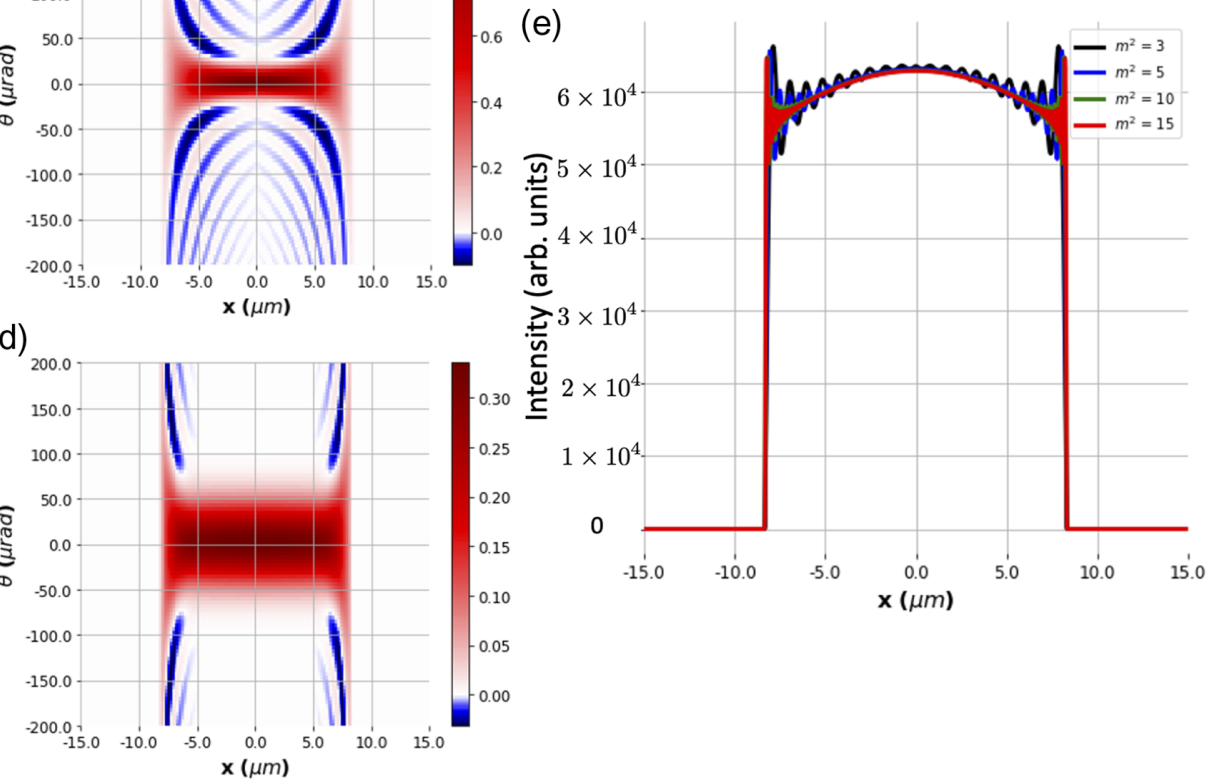

FIG. 4. WFD plots for Gaussian beam after passing through single slit aperture. Beam size, $\sigma_{x}$, has been fixed and divergence varies according to the parameter $m^{2}$ in Eq. (35). The radiation wavelength $\lambda=3.98 \AA$. (a) $m^{2}=3, \sigma_{\theta}=5.90 \mu \mathrm{rad}$ (b) $m^{2}=5, \sigma_{\theta}=$ $9.83 \mu \mathrm{rad}$ (c) $m^{2}=10, \sigma_{\theta}=19.66 \mu \mathrm{rad}$ (d) $m^{2}=15, \sigma_{\theta}=29.49 \mu \mathrm{rad}$ (e) projection on spatial axis for cases (a)-(d).

numerical result must be redeposited onto the initial grid, one tends to truncate the calculation within a smaller range. The WFD was computed numerically by first constructing a discrete Gaussian along with discrete representation of the single slit aperture WFD. These two functions were then convolved in $\theta$ to compute the result following the aperture. Finally, this WFD was drifted for $10 \mathrm{~cm}$ by application of the drift transfer matrix and redeposition

(a)

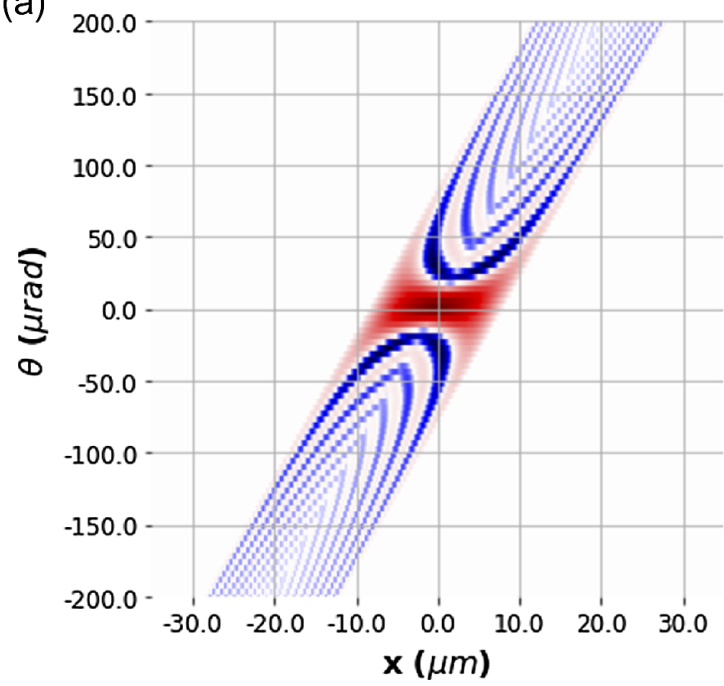

upon the original grid using the method as first reported in [29].

Figure 7 displays the dependence of degree of coherence on increasing $m$ value both before and after the single slit aperture. We show agreement between numerical and analytical [Eq. (41)] calculations for this quantity before the aperture. We note that following propagation through the aperture, the degree of coherence has increased which is

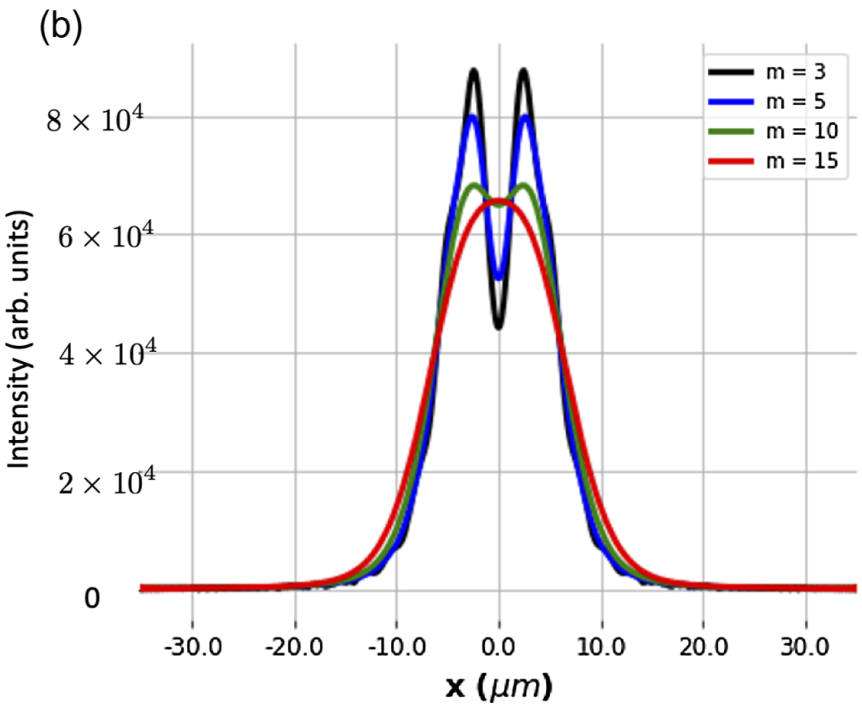

FIG. 5. (a) WFD from Figure 4 for $m^{2}=3$ after drifting $10 \mathrm{~cm}$. (b) Spatial projections of drifted WFDs for $m^{2}=3,5,10$ and 15 . 


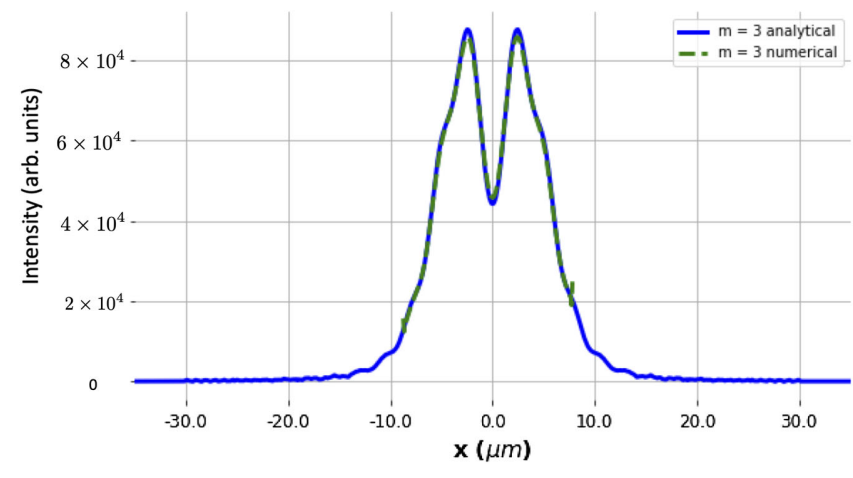

FIG. 6. Comparison of analytically and numerically calculated spatial projections of drifted WFDs for $\mathrm{m}=3$.

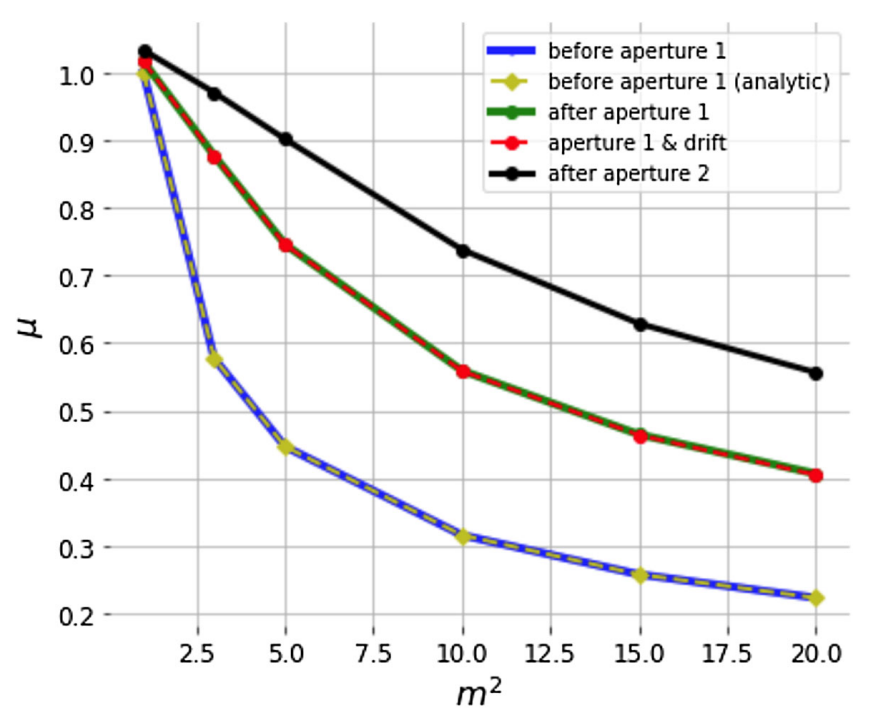

FIG. 7. Degree of coherence with varying $m$ values before and after the aperture.

to be expected since a more coherent subset of the radiation has been selected by the aperture. In addition, we confirm that the degree of coherence is invariant under the final free space propagation which is predicted by Eq. (23).

\section{EXAMPLES-UNDULATOR RADIATION}

\section{A. Partially coherent undulator radiation}

As an example of transporting a partially coherent Wigner function, we will consider the case of undulator radiation resulting from a beam of electrons in a synchrotron light source.

A single electron with initial phase space coordinates $\vec{z}_{0}$ passing through the undulator will produce a coherent wavefront $E_{u}(x)$. The electron will be drawn from a distribution of electrons, $f_{e}\left(\vec{z}_{0}\right)$, that are circulating in the electron storage ring. This distribution generally takes on a Gaussian form resulting from an equilibrium between the damping and diffusion effects from synchrotron radiation [30,31]. Due to differences in the longitudinal coordinates of the emitting electrons, the radiated wavefronts will add incoherently and produce partially coherent radiation.

We assume that the radiation will satisfy

$$
W\left(\vec{z} ; \vec{z}_{0}\right)=\mathcal{W}\left(E\left(\vec{x} ; \vec{z}_{0}\right)\right)=W_{0}\left(\vec{z}-\vec{z}_{0}\right)
$$

where

$$
W_{0}(\vec{z})=\mathcal{W}\left(E\left(\vec{x} ; \vec{z}_{0}=\overrightarrow{0}\right)\right) .
$$

Under these conditions, the multielectron Wigner function $W_{m e}$ may be related to the single electron Wigner function via convolution with the electron beam distribution:

$$
W_{m e}(\vec{z})=W_{s e}(\vec{z}) * f_{e}(\vec{z}) .
$$

Next we note that the convolution of the single electron Wigner function with the electron beam distribution may be postponed until the first aperture by applying the following identity:

$$
U_{M_{1}} W_{s e}(\vec{z}) * f_{e}(\vec{z})=W_{s e}\left(M_{1} \vec{z}\right) * f_{e}(\vec{z}),
$$

where the transfer matrix, $M_{1}$, represents the propagation from the source to the first aperture. Recall the discussion in Sec. II D for the definition of the operator $U_{M_{1}}$. This identity is known as the "emittance convolution theorem" attributed to K.-J. Kim (see e.g. discussion in [32] and references therein). This theorem allows us to propagate a single coherent wavefront until the first aperture where we then need to construct the partially coherent Wigner function via convolution with the electron beam phase space distribution that has been propagated to the same position via the transfer matrix of the first section $M_{1}$. The fact that apertures are represented by only a partial convolution prevents us from extending this identity and applying the convolution beyond the first aperture.

As an example of UR, we consider the undulator and electron beam with parameters defined in Table I. The software package synchrotron radiation workshop (SRW) $[33]^{9}$ is used for the initial wavefront calculation that will be used to construct the corresponding WFD. We compute the radiation at the first optical element and progress it through the lens and drift such that we achieve the one-toone focusing yielding the radiation as it would appear at the center of the undulator. Figure 8(a) and (b) display the real and imaginary parts of the electric field and in (c) and (d) we have projected these fields onto the horizontal axis and normalized them according to Eq. (B2). Note that since our planar undulator has a vertical magnetic field, we have

\footnotetext{
${ }^{9}$ The SRW source code is maintained by Oleg Chubar and is avaialble at https://github.com/ochubar/srw
} 

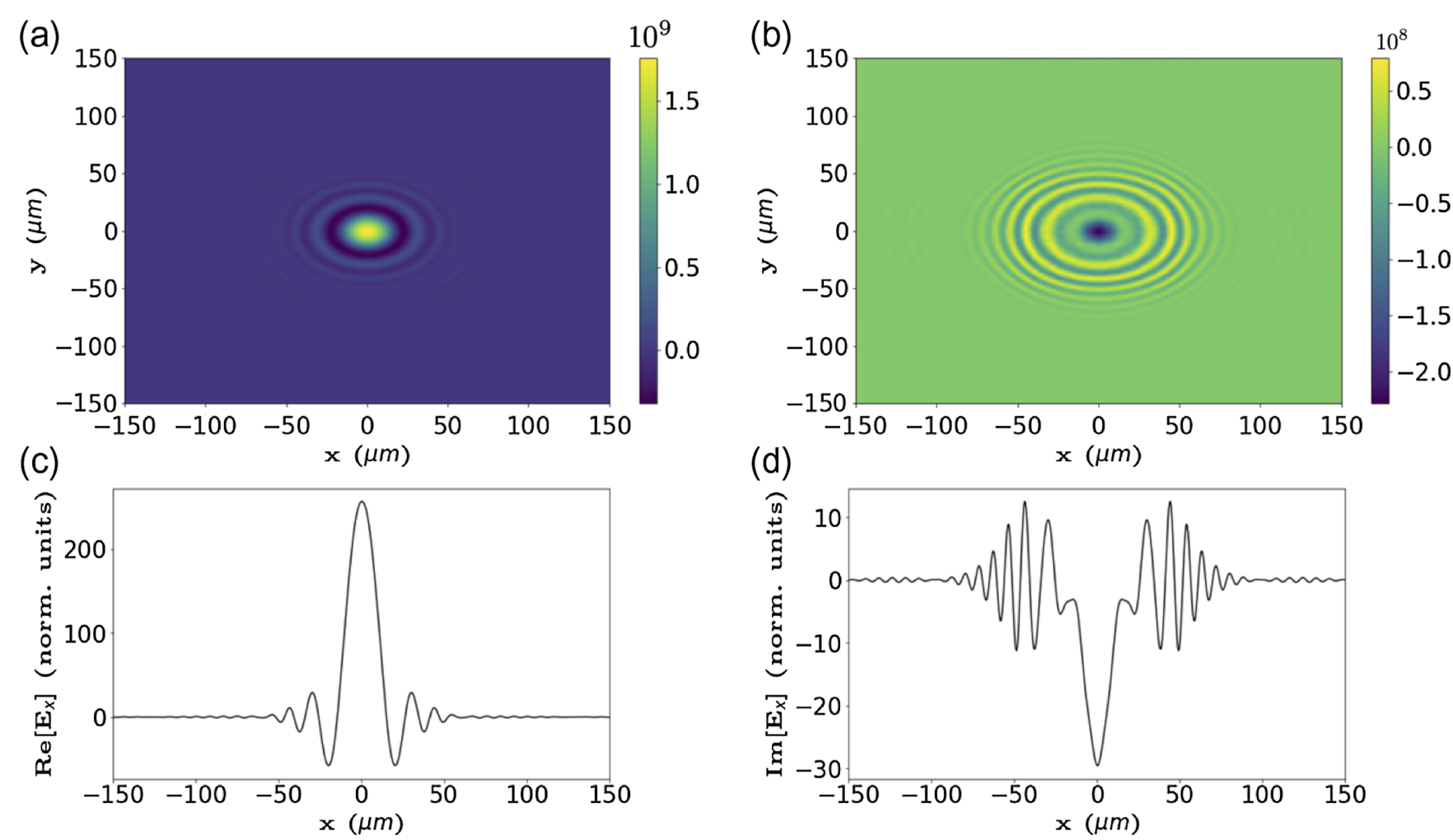

FIG. 8. Undulator radiation at first harmonic energy of $3.115 \mathrm{keV}$. (a) Real part of electric field. (b) Imaginary part of electric field. (c) Horizontal projection of real electric field. (d) Horizontal projection of imaginary electric field.

selected the dominant polarization component which is horizontal.

The UR WFD is constructed from the electric field projections and is displayed in Fig. 9(a). Figure 9(b) displays the electron beam distribution corresponding to the APS Upgrade parameters given in Table I. The convolution of the single electron UR WFD and the electron beam distribution results in the multi-electron WFD given in Fig. 9(c). In this case, we can see that we are near the diffraction limit as the electron beam size is of the same order as the UR single electron radiation beam size. The finite emittance result shows properties of both the underlying UR WFD and the Gaussian electron beam distribution. ${ }^{10}$ This WFD is then propagated through the example beam line and the results from both our WFD transport method and SRW are detailed in the following section.

\section{B. Comparison with multielectron SRW simulation}

We now report the results from transporting the UR through the single slit aperture and subsequent drift. The

\footnotetext{
${ }^{10} \mathrm{We}$ note that this result is dependent on the undulator radiation energy used of $3.115 \mathrm{keV}$. Higher energy radiation will take up a smaller phase space footprint, and the electron beam contribution will be correspondingly larger. It is thus easier to reach the diffraction limit of high coherence for lower energy radiation, for a fixed electron beam size and divergence.
}

multi-electron WFD is computed by convolution with the electron beam and propagation through the single slit aperture is performed by means of convolution in $\theta$ with the aperture WFD. The free space drift is performed as done in the Gaussian case with the same linear transport algorithm used in the preceeding section. We note that this transport algorithm may be applied to any WFD.

This numerical result was benchmarked against a partially coherent SRW calculation wherein the same example beam line was used. The multi-electron SRW calculation requires running the coherent calculation many times for different macro-electrons. In this case of $42 \mathrm{pm}$ emittance, we found we could achieve convergence with 5000 macroparticles. Larger emittance, such as $4 \mathrm{~nm}$ requires larger numbers of macro-electrons: up to 50,000. The results shown here for $42 \mathrm{pm}$ used the more conservative value of 50,000 macro-electrons, though this was not strictly required.

These results are shown in Fig. 10. Figure 10(a) displays the final numerically computed WFD. We note similarity to the Gaussian case being dominated by the structure of the single slit aperture Wigner filter function. In Fig. 10(b), the spatial projections of the final WFD have been calculated and used to benchmark our numerical method against the computationally intensive multielectron SRW calculation. The partial coherence reflects the effect of the electron beam distribution which, as mentioned, has been drawn from the APS Upgrade beam parameters. We note that the 

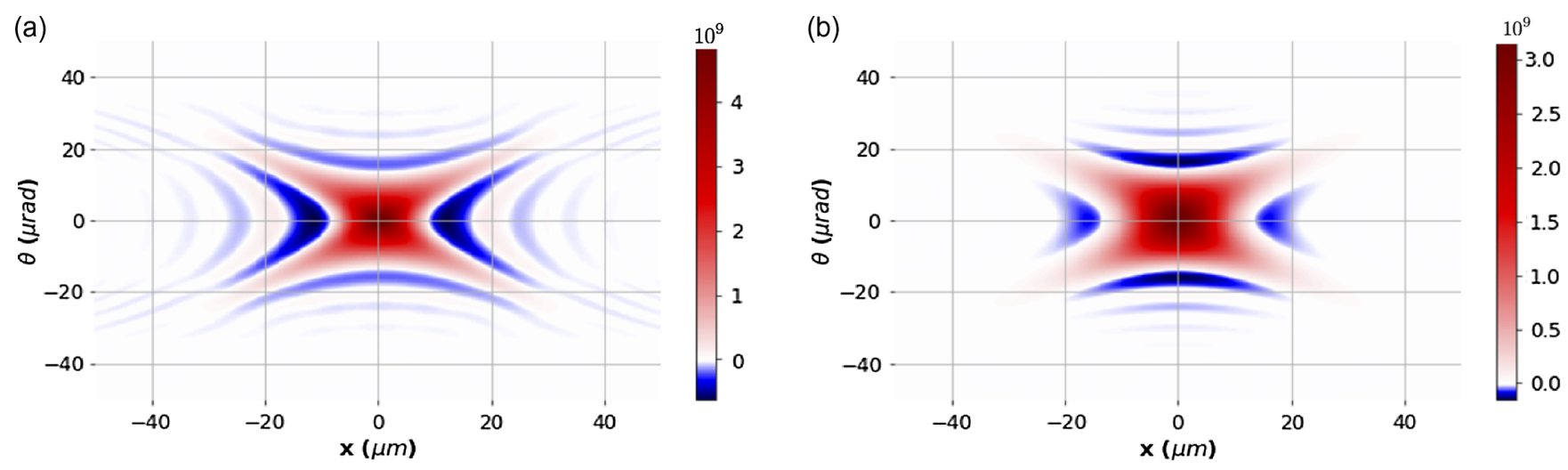

(c)
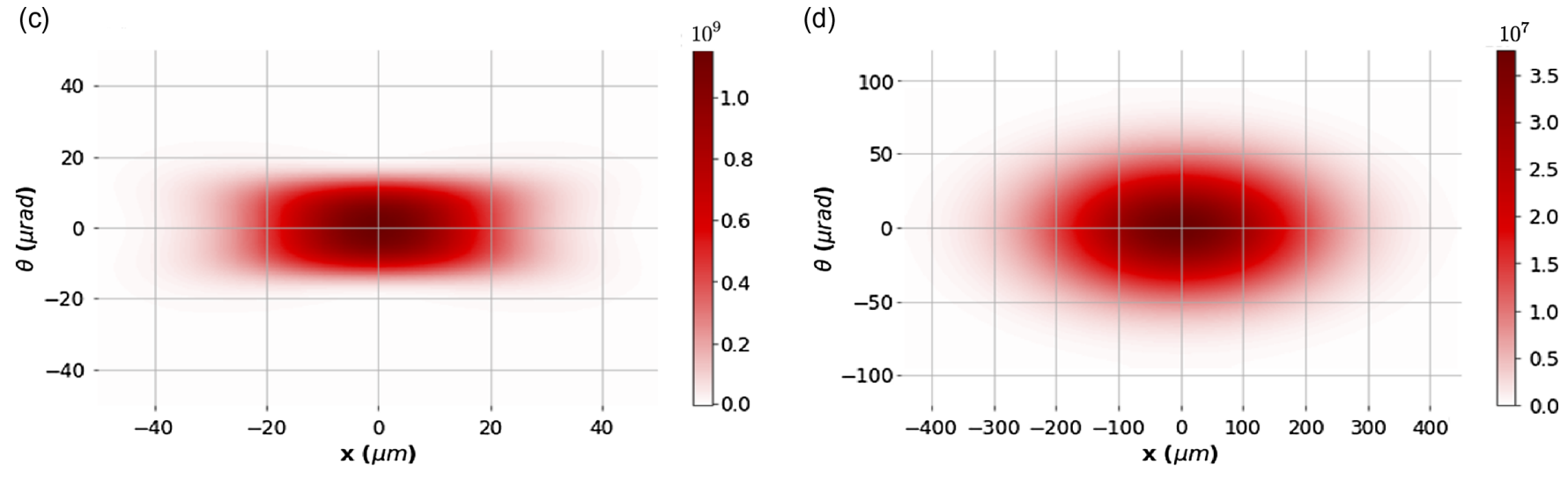

FIG. 9. Undulator radiation multielectron WFD for varying emittance $\epsilon$ : (a) zero emittance (fully coherent) (b) 4 pm (c) $40 \mathrm{pm}$ (d) $4 \mathrm{~nm}$.
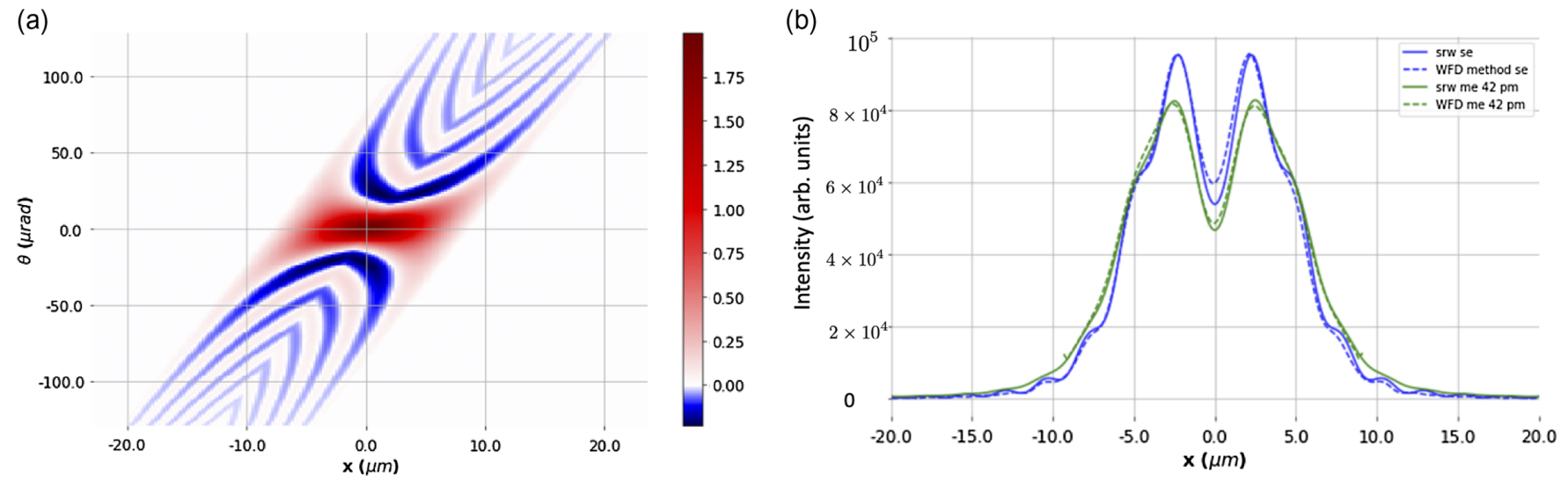

FIG. 10. (a) Fully coherent UR WFD following propagation through single slit aperture and $10 \mathrm{~cm}$ drift. (b) Comparison between WFD method and SRW calculation for fully coherent, single electron (se), and partially coherent, multielectron (me), UR spatial projections.

$42.2 \mathrm{pm}$ emmittance electron beam, if representing directly a Gaussian WFD, would correspond to an $m^{2}$ value of 1.33 . Recall Eq. (35). Figure 11 provides a comparison between the propagated Gaussian and undulator radiation for the cases of fully and partially coherent radiation. For the Gaussian radiation, the second moments have been taken from adding in quadrature the undulator $\sigma_{x}$ and $\sigma_{\theta}$ with those of the electron beam. We note that for the fully coherent case (single electron), there is not perfect agreement between the two calculations, with the Gaussian case having a larger central dip from diffraction. In the case of the $42 \mathrm{pm}$ emittance, however, the Gaussian result quite adequately reproduces the more complex undulator radiation calculation. ${ }^{11}$

\footnotetext{
${ }^{11}$ For purposes of speed comparison of the two methods for partially coherent propagation, one should also use the method of coherent mode decomposition [8].
} 


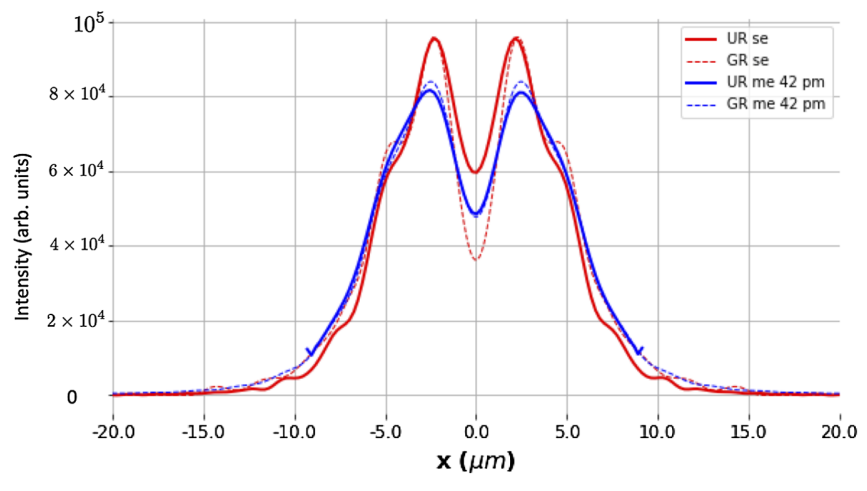

FIG. 11. Spatial projections of Gaussian and undulator radiation propagated through single slit aperture and $10 \mathrm{~cm}$ drift via WFD method. In the legend, se denotes single electron (fully coherent) and me denotes multi-electron (partially coherent). For the latter, the photon beam has been convolved with the APS-U electron beam given in Table I.

\section{COMMENTS ON COMPUTATIONAL COMPLEXITY}

In this paper, we have proposed direct computation of the partially coherent synchrotron radiation Wigner function, followed by evolution through a simplified (matrixaperture) beam line represented by linear transport sections divided by physical apertures. Exact statements about computational complexity are challenging, but we try to lay out a few of the relevant issues in comparing to alternate approaches to such computations.

Let us assume that a model is set up in a wave optics modeling code, using SRW as our example. A Gaussian electron beam with known parameters is determined from the given electron storage ring finding equilibrium due to synchrotron radiation and other physical effects such as intrabeam scattering. Alternatively, in the context of an online model, beam measurements can determine the electron beam Gaussian parameters. Next, given the magnetic field of the undulator insertion device, the coherent wavefront may be computed using the integral method contained in the SRW code. So far the method of this paper and the standard SRW method are equivalent.

Let us assume that the electric field is computed on a grid of size $N_{x} \times N_{y}$. First, consider the coherent SRW computations [34]. A drift space propagation involves a Fresnel transform in the near field and a Fraunhofer transform in the far field. This represents either 2 or 1 FFTs, respectively [35], and so we take the computation complexity to be $\mathcal{O}\left(N_{x} N_{y} \log \left(N_{x}\right) \log \left(N_{y}\right)\right)$. A thin lens element is propagated via multiplication by a quadratic phase factor, a so-called "chirp multiplication". This is an $\mathcal{O}\left(N_{x} N_{y}\right)$ operation. In the general context of the matrix-aperture beam lines considered in this paper, we may decompose the linear transport sections into a sequence of thin lenses and drifts (see eg. Ref. [36]). Likewise, an aperture propagation is also just a multiplication in terms of the electric field, and is thus also $\mathcal{O}\left(N_{x} N_{y}\right)$.

The method used for partial coherence in SRW involves sampling of the electron beam distribution and performing a coherent propagation for each macroparticle. Thus, for $N_{m}$ macroparticles, this factor is to be multiplied to determine the overall algorithmic complexity.

In contrast, the method we propose here involves a single propagation, and thus, if the Wigner function propagation were comparable to the coherent propagation, there would be a gain by a factor of $N_{m}$ in the computational speed. However, there are additional considerations with our algorithm. First, the grid size is increased due to the functions being defined on phase space. In particular, for $4 \mathrm{D}$, the grid size is now $N_{x}^{2} \times N_{y}^{2}$. The Wigner function transport algorithm involves first a matrix multiplication for each grid point and then the redeposition onto the original grid. This method is described in Ref. [37]. The matrix multiplication gives a complexity of the number of grid points $N_{x}^{2} \times N_{y}^{2}$. The deposition involves solving of a linear equation of the form $L y=A x$ where the matrices, $L$ and $A$ are known, along with the vector of weights $x$.

Thus, the algorithmic complexity comes from redepositing the Wigner space coordinates to the grid, which is akin to charge deposition in electrostatic particle-in-cell algorithms, and from the sparse matrix multiplication. Charge deposition to a grid of $N_{x} \times N_{y}$ cells is not a memory-local operation. The matrices this algorithm produces are $N_{x}^{4} \times$ $N_{y}^{4}$ in size, and one of the matrices is sparse. Because this is a matrix-vector multiplication, the algorithmic complexity comes from two matrix-vector products of complexity $\mathcal{O}\left(N_{x}^{4} \times N_{y}^{4}\right)$. Our implementation is a brute force approach, although more sophisticated methods could bring the computation time down much further.

\section{CONCLUSION}

We have described a new, unified method for transporting coherent and partially coherent radiation through a beam line of linear optical elements and apertures. This approach relies on transporting the Wigner distribution of the radiation wavefront in a manner akin to single-particle tracking in particle accelerators. In contrast with physical optics modeling, the formalism is the same for fully coherent and partially coherent radiation, and has the same computational time and complexity. The matrices in the matrix-aperture beam line we have defined may be computed with ray tracing, in the general case, or analytically for simplified beam line models. We thus show how ray tracing may be unified with wave optics, at least within this linear approximation.

We have demonstrated this approach by transporting both a Gaussian wave front and an undulator radiation wavefront through drift space and a single slit aperture. In the case of the Gaussian wave front, we find excellent 
agreement between an analytical result, the Wigner distribution approach, and a the well-established SRW physical optics code. For the undulator radiation, we have excellent agreement between the Wigner distribution approach and multi-electron SRW calculations. Finally, we have seen that for large enough emittance, the Gaussian results approximate the undulator radiation to a good degree of accuracy.

Future work will extend this technique to nonlinear optical elements, which will allow us to account for optical aberrations. We have briefly described the formalism for nonlinear optical elements in Appendix C. Extension to the nonlinear case will raise new issues, for future exploration. See e.g. [38] for some understanding of the potential resulting complications.

\section{ACKNOWLEDGMENTS}

The authors would like to thank A. Wojdyla for valuable discussion and for alerting them to the literature on Linear Canonical Transforms, including Ref. [39]. This material is based upon work supported by the U.S. Department of Energy, Office of Science, Office of Basic Energy Sciences, under Awards No. DE-SC0020593 and No. DESC0018571.

\section{APPENDIX A: COHERENT WAVE OPTICS}

Here we describe, for completeness, the approach used for propagating a radiation wavefront through a beam line using physical optics techniques. The propagation of this wavefront through an optical beam line is the goal of physical optics software such as SRW and XRT [40,41]. In these codes, there exist a variety of numerical implementations of propagators to transport the wavefront through free space, lenses, mirrors, gratings, and other optical elements.

We begin with a complex electric field $E_{0}(x, y, s=0)$ at the entrance of the beam line. Optical transport beam lines in synchrotron radiation facilities can often be represented with the scalar paraxial optics approximation $[42,43]$. For example, the near-field Fresnel integral for free-space propagation can be written as a convolution:

$$
E(x, y, s)=E_{0}(x, y) * h(x, y, s),
$$

with

$$
h(x, y, s)=\frac{e^{i k s}}{i \lambda s} e^{i \frac{k}{22}\left(x^{2}+y^{2}\right)} .
$$

A thin lens may be traversed via

$$
E\left(x, y, s^{\prime}\right)=e^{-i \frac{k}{2 f}\left(x^{2}+y^{2}\right)} E(x, y, s),
$$

with $k=\frac{2 \pi}{\lambda}, n$ is the index of refraction, and $f$ is the focal length [44].

The combination of drifts, lenses, and focusing mirrors together can be combined to create a symplectic transport matrix $M$ for the geometric ray optics as given in Eq. (15). Knowing $M(s)$, one may propagate the wavefront through the linear beam line down the channel via linear canonical transformation (LCT) [39]. Explicitly for 4D phase space, the transfer matrix is written in the form

$$
M=\left(\begin{array}{ll}
A & B \\
C & D
\end{array}\right) .
$$

The transformed electric field $E_{f}(\vec{x})$ is given by

$$
E_{f}(\vec{x})=\frac{1}{\sqrt{\operatorname{det}(i B)}} \int e^{i \pi \vec{u}^{T} \mathcal{M} \vec{u}} E_{i}(\vec{x}) d \vec{x}_{i}
$$

with

$$
\vec{u}=\left(\begin{array}{ll}
\vec{x}_{f} & \vec{x}_{i}
\end{array}\right)
$$

and

$$
\mathcal{M}=\left(\begin{array}{ll}
D B^{-1} & -B^{-1} \\
-B^{-1} & B^{-1} A
\end{array}\right)
$$

where the subscripts $f$ and $i$ represent initial and final.

We point out here, that in addition to use of analytical expressions for beam line elements to determine the Hamiltonian, and thus find the transfer matrix $M(s)$, one may also use a ray tracing code, set up the beam line, and by tracking a series of rays offset from the central trajectory, derive the transport matrix numerically along the beam line.

The effect of the apertures, represented by the transfer functions $t_{j}(x, y)$ on the wavefront simply by multiplication:

$$
E\left(x, y ; s^{\prime}\right)=t_{j}(x, y) E(x, y ; s) .
$$

\section{APPENDIX B: NORMALIZATION OF WAVEFRONTS AND WIGNER FUNCTIONS}

In this paper, we will assume electric fields which satisfy the separability condition

$$
E(x, y ; s)=E_{0} E_{x}(x ; s) E_{y}(y ; s),
$$

where $E_{0}$ is a constant with units of electric field.

We normalize the separate electric field components in 1-D such that

$$
\int_{-\infty}^{\infty} E^{*}(x) E(x) d x=1
$$




$$
\int_{-\infty}^{\infty} E^{*}(\theta) E(\theta) d \theta=1
$$

Following Bazarov [15], we have normalized the electric field in the same way as wave functions are normalized in quantum mechanics. The second moments of the field distribution in coordinate and angular representations may now be calculated as

$$
\begin{aligned}
& \left\langle x^{2}\right\rangle=\int_{-\infty}^{\infty} x^{2} E^{*}(x) E(x) d x, \\
& \left\langle\theta^{2}\right\rangle=\int_{-\infty}^{\infty} \theta^{2} E^{*}(\theta) E(\theta) d \theta .
\end{aligned}
$$

We now introduce the Wigner function defined from the electric field, $E(x)$, as follows

$W(x, \theta)=\frac{1}{\lambda} \int_{-\infty}^{\infty} E^{*}\left(x-\frac{\phi}{2}\right) E\left(x+\frac{\phi}{2}\right) e^{\frac{-2 \pi i}{\lambda} \phi \theta} d \phi$,

where $W(x, \theta)$ will be normalized as

$$
\int_{-\infty}^{\infty} \int_{-\infty}^{\infty} W(x, \theta) d x d \theta=1 .
$$

The Wigner function can be thought of as a probability distribution in phase space except for the fact that it may become negative. The second moments are given simply as

$$
\begin{aligned}
& \left\langle x^{2}\right\rangle=\int_{-\infty}^{\infty} \int_{-\infty}^{\infty} x^{2} W(x, \theta) d x d \theta, \\
& \left\langle\theta^{2}\right\rangle=\int_{-\infty}^{\infty} \int_{-\infty}^{\infty} \theta^{2} W(x, \theta) d x d \theta, \\
& \langle x \theta\rangle=\int_{-\infty}^{\infty} \int_{-\infty}^{\infty} x \theta W(x, \theta) d x d \theta .
\end{aligned}
$$

\section{APPENDIX C: TRANSPORT OF WIGNER FUNCTION UNDER NONLINEAR MAPS}

For propagation of the Wigner function, Bazarov (Property 5) uses the Hamiltonian $\mathcal{H}=\frac{\hat{p}^{2}}{2 m}+V(x)$ for the quantum mechanics case of a particle in a potential. We have used a different Hamiltonian for the optics case. The problem of general transport of the Wigner function remains. The evolution equation for the Wigner function under a general Hamiltonian is given as follows [13]

$$
\frac{\partial W\left(x, \theta_{x}, y, \theta_{y} ; s\right)}{\partial s}=[W, H]_{*},
$$

where the Moyal bracket is defined for arbitrary phase space functions $f$ and $g$ as

$$
[f, g]_{*}=\frac{1}{i \chi}(f * g-g * f),
$$

and the Moyal star is given by

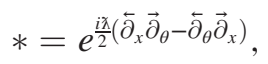

with the arrows representing action of the derivative, either to the left or right, depending on arrow orientation.

\section{APPENDIX D: DERIVATION OF SINGLE SLIT APERTURE WIGNER FILTER}

Here we derive the Wigner function for a single slit aperture. The transfer function is given by

$$
t(x)=\operatorname{rect}(x) \equiv \Pi(x),
$$

where we have used the symbol $\Pi$ for the rect function for ease of notation. Applying the definition of the Wigner function, we find

$W(x, \theta)=\frac{1}{\lambda} \int_{\infty}^{\infty} \Pi\left(\frac{x-\frac{\phi}{2}}{a}\right) \Pi\left(\frac{x+\frac{\phi}{2}}{a}\right) \cos \left(\frac{2 \pi i}{\lambda} \phi \theta\right) d \phi$,

where we have taken the real part of the complex exponential since the result must be real. Consideration of the geometry of the integration region and the product of the rect functions allows us to write this integral as follows:

$$
W(x, \theta)=\Pi(y) \frac{2 a}{\lambda} \int_{|y|-\frac{1}{2}}^{\frac{1}{2}-|y|} \cos \left(\frac{2 \pi}{\lambda}(2 a \psi \theta)\right) d \psi,
$$

where we have defined $y=\frac{x}{a}$. We now do the integration of the cos function over the finite domain, and after some simplification, we arrive at

$$
W(x, \theta)=\Pi\left(\frac{x}{a}\right) \frac{1}{\pi} \sin \left(\frac{2 \pi \theta}{\lambda}(a-2|x|)\right) .
$$

\section{APPENDIX E: ANALYTICAL CALCULATION OF A GAUSSIAN WIGNER DISTRIBUTION THROUGH A SINGLE SLIT}

The passage of a Gaussian Wigner distribution through a slit can be evaluated in terms of the integral

$$
\mathcal{I}(x, \theta)=\int_{-\infty}^{\infty} d \tau \frac{\sin \tau q}{\tau} \exp \left\{-\frac{(\theta-\tau)^{2}}{2 \sigma_{\theta}^{2}}\right\} .
$$

It is convenient to normalize all the variables to $\sigma_{\theta}$, so that $\hat{\theta}=\theta / \sigma_{\theta}, \hat{\tau}=\tau / \sigma_{\theta}$, and $\hat{q}=\sigma_{\theta} q$, so that the integral becomes: 


$$
\mathcal{I}(x, \theta)=\int_{-\infty}^{\infty} d \hat{\tau} \frac{\sin \hat{\tau} \hat{q}}{\hat{\tau}} \exp \left\{-\frac{1}{2}(\hat{\theta}-\hat{\tau})^{2}\right\}
$$

This integral can be rewritten as the imaginary part of an indefinite integral with respect to $q$,

$$
\mathcal{I}(x, \theta)=\operatorname{Im}\left[i \int d \hat{q} \int_{-\infty}^{\infty} d \hat{\tau} e^{i \hat{\tau} \hat{q}} \exp \left\{-\frac{1}{2}(\hat{\theta}-\hat{\tau})^{2}\right\}\right] .
$$

Expanding the Gaussian argument brings a Gaussian $\theta$ envelope out front:

$$
\begin{aligned}
\mathcal{I}(x, \theta)= & e^{-\frac{\hat{\theta}^{2}}{2}} \operatorname{Im}\left[i \int d \hat{q} \int_{-\infty}^{\infty} d \hat{\tau}\right. \\
& \left.\times \exp \left\{-\frac{1}{2}\left(\hat{\tau}^{2}+2 i \hat{\tau}(\hat{q}+i \hat{\theta})\right)\right\}\right] .
\end{aligned}
$$

The argument can be simplified by completing the square, noting that

$$
\hat{\tau}^{2}+2(\hat{\theta}+i \hat{q}) \hat{\tau}=(\hat{\tau}+\hat{\theta}+i \hat{q})^{2}-(\hat{\theta}+i \hat{q})^{2},
$$

which then gives the integral as

$$
\begin{aligned}
\mathcal{I}(x, \theta) & =e^{-\frac{\hat{\theta}^{2}}{2}} \operatorname{Im} \\
& \times[i \int d \hat{q} e^{\frac{1}{2}(\hat{\theta}+i \hat{q})^{2}} \underbrace{\int_{-\infty}^{\infty} d \hat{\tau} \exp \left\{-\frac{1}{2}\left(\hat{\tau}^{2}+i \hat{q}+\hat{\theta}\right)\right\}}_{\sqrt{2 \pi}}],
\end{aligned}
$$

and the integral becomes

$$
\mathcal{I}(x, \theta)=\sqrt{2 \pi} e^{-\frac{\hat{\theta}^{2}}{2}} \operatorname{Im}\left[i \int d \hat{q} e^{\frac{1}{2}(\hat{\theta}+i \hat{q})^{2}}\right],
$$

and which is then given by

$$
\mathcal{I}(x, \theta)=\sqrt{\frac{\pi}{2}} \operatorname{Im}\left[i \operatorname{erf}\left(\frac{\hat{q}+i \hat{\theta}}{\sqrt{2}}\right)\right] .
$$

[1] M. S. del Rio, R. Celestre, M. Glass, G. Pirro, J. R. Herrera, R. Barrett, J.C. da Silva, P. Cloetens, X. Shi, and L. Rebuffi, A hierarchical approach for modeling X-ray beamlines: application to a coherent beamline, J. Synchrotron Radiat. 26, 1887 (2019).

[2] X. Shi, R. Reininger, R. Harder, and D. Haeffner, Development of partially-coherent wavefront propagation simulation methods for 3rd and 4th generation synchrotron radiation sources, in Proceedings of SPIE, Advances in Computational Methods for X-Ray Optics IV, Vol. 10388 (SPIE, Bellingham, Washington USA, 2017) p. 103880C, https://doi.org/10.1117/12.892812.

[3] M. S. del Rio, N. Canestrari, F. Jiang, and F. Cerrina, SHADOW3: a new version of the synchrotron X-ray optics modelling package, J. Synchrotron Radiat. 18, 708 (2011).

[4] O. Chubar and P. Elleaume, Accurate and efficient computation of synchrotron radiation in the near field region, Conf. Proc. C980622, 1177 (1998), https://inspirehep.net/ literature/487818.

[5] J. W. Goodman, Statistical Optics, 2nd ed. (Wiley, New York, 2015).

[6] G. Geloni, E. Saldin, E. Schneidmiller, and M. Yurkov, Transverse coherence properties of X-ray beams in thirdgeneration synchrotron radiation sources, Nucl. Instrum. Methods Phys. Res., Sect. A 588, 463 (2008).

[7] O. Chubar, L. Berman, Y. S. Chu, A. Fluerasu, S. Hulbert, M. Idir, K. Kaznatcheev, D. Shapiro, Q. Shen, and J. Baltser, Development of partially-coherent wavefront propagation simulation methods for 3rd and 4th generation synchrotron radiation sources, Adv. Comput. Methods X-Ray Opt. II 8141, 814107 (2011).

[8] M. Glass and M. S. del Rio, Coherent modes of X-ray beams emitted by undulators in new storage rings, Europhys. Lett. 119, 34004 (2017).

[9] R. Li and O. Chubar, in Advances in Computational Methods for X-Ray Optics V, Vol. 11493 (SPIE, Bellingham, Washington USA, 2020) p. 114930G.

[10] R. Coïsson and S. Marchesini, Gauss-schell sources as models for synchrotron radiation, J. Synchrotron Radiat. 4, 263 (1997).

[11] K.-J. Kim, in Insertion Devices for Synchrotron Sources, Vol. 0582, edited by I.E. Lindau and R. O. Tatchyn, International Society for Optics and Photonics (SPIE, New York, 1986) pp. 2-9.

[12] T. L. Curtright, D. B. Fairlie, and C. K. Zachos, Quantum Mechanics in Phase Space: An Overview with Selected Papers (World Scientific, Singapore, 2005).

[13] T. L. Curtright and C. K. Zachos, Quantum mechanics in phase space, Asia Pacific Physics Newsletter 01, 37 (2012).

[14] E. Wigner, On the quantum correction for thermodynamic equilibrium, Phys. Rev. 40, 749 (1932).

[15] I. V. Bazarov, Synchrotron radiation representation in phase space, Phys. Rev. Accel. Beams 15, 050703 (2012).

[16] A. E. Siegman, Lasers (University Science Books, Mill Valley, CA, 1986).

[17] C. Ferrero, D.-M. Smilgies, C. Riekel, G. Gatta, and P. Daly, Extending the possibilities in phase space analysis of synchrotron radiation x-ray optics, Appl. Opt. 47, E116 (2008).

[18] T. Tanaka, Numerical methods for characterization of synchrotron radiation based on the Wigner function method, Phys. Rev. Accel. Beams 17, 060702 (2014).

[19] I. A. Vartanyants and A. Singer, Coherence properties of hard $\mathrm{x}$-ray synchrotron sources and $\mathrm{x}$-ray free-electron lasers, New J. Phys. 12, 035004 (2010).

[20] L. Mandel and E. Wolf, Optical Coherence and Quantum Optics (Cambridge University Press, Cambridge, England, 1995). 
[21] A. J. Dragt, Lie algebraic theory of geometrical optics and optical aberrations , J. Opt. Soc. Am. 72, 372 (1982).

[22] A. Dragt, "Lie algebraic methods for ray and wave optics," (1995), unpublished.

[23] B. Nash, N. Goldring, J. Edelen, C. Federer, P. Moeller, and S. Webb, in Advances in Computational Methods for $X$-Ray Optics V, Vol. 11493 (SPIE, Bellingham, Washington USA, 2020) p. 114930C.

[24] C. J. Román-Moreno, R. Ortega-Martínez, and C. FloresArvizo, The Wigner function in paraxial optics II. Optical diffraction pattern representation, Revista Mexicana de Física 49, 290 (2003), https://rmf.smf.mx/ojs/rmf/article/ view/3166.

[25] R. P. Walker, Undulator radiation brightness and coherence near the diffraction limit, Phys. Rev. Accel. Beams 22, 050704 (2019).

[26] R. Khubbutdinov, A. P. Menushenkov, and I. A. Vartanyants, Coherence properties of the high-energy fourth-generation X-ray synchrotron sources, J. Synchrotron Radiat. 26, 1851 (2019).

[27] B. Nash, O. Chubar, N. Goldring, D. L. Bruhwiler, P. Moeller, R. Nagler, M. Rakitin, S. Gwo, D.-J. Huang, and D.-H. Wei, Detailed x-ray brightness calculations in the sirepo GUI for SRW, AIP Conf. Proc. 2054, 060080 (2019).

[28] B. Nash, O. Chubar, D. L. Bruhwiler, M. Rakitin, P. Moeller, R. Nagler, and N. Goldring, in Advances in Laboratory-based X-Ray Sources, Optics, and Applications VII, Vol. 11110 (SPIE, Bellingham, Washington USA, 2019) p. 111100K.

[29] B. Nash, J. Edelen, N. Goldring, and S. Webb, in 13th International Computational Accelerator Physics Conference ICAP'18 (Michigan State University, East Lansing, Michigan, USA, 2019) p. TUPAG17.

[30] B. Nash, J.-H. Wu, and A. W. Chao, Equilibrium beam distribution in an electron storage ring near linear synchrobetatron coupling resonances, Phys. Rev. ST Accel. Beams 9 (2006), 032801.

[31] M. Sands, The physics of electron storage rings: An introduction, Conf. Proc. C 6906161, 257 (1968), https://inspirehep.net/literature/60854.
[32] M. Glass, Statistical optics for synchrotron emission: numerical calculation of coherent modes, Ph.D. thesis, Université Grenoble Alpes (2017).

[33] O. Chubar, A. Fluerasu, L. Berman, K. Kaznatcheev, and L. Wiegart, Wavefront propagation simulations for beamlines and experiments with "Synchrotron Radiation Workshop", J. Phys. Conf. Ser. 425, 162001 (2013).

[34] O. V. Chubar, Precise computation of electron-beam radiation in nonuniform magnetic fields as a tool for beam diagnostics, Rev. Sci. Instrum. 66, 1872 (1995).

[35] O. Chubar and R. Celestre, Memory and CPU efficient computation of the Fresnel free-space propagator in Fourier optics simulations, Opt. Express 27, 28750 (2019).

[36] S.-C. Pei and S.-G. Huang, Fast discrete linear canonical transform based on CM-CC-CM decomposition and FFT, IEEE Trans. Signal Process. 64, 855 (2015).

[37] J. P. Edelen and S. D. Webb, A vlasov algorithm derived from phase space conservation, arXiv:2007.12227.

[38] A. J. Dragt and S. Habib, How wigner functions transform under symplectic maps, arXiv:quant-ph/9806056.

[39] J. Healy, M. Kutay, H. Ozaktas, and J. Sheridan, Linear Canonical Transforms: Theory and Applications, Vol. 198 (Springer, New York City, NY, USA, 2016).

[40] “xrt (XRayTracer)—xrt 1.3.3 documentation," (2020), [Online; accessed 23. Apr. 2020].

[41] R. Chernikov and K. Klementiev, Recent progress of the XRT: ray tracing and wave propagation toolkit, Proceedings of SPIE, Advances in Computational Methods for X-Ray Optics IV 10388, 1038806 (2017), https://doi.org/10.1117/ 12.2273430.

[42] J. Bahrdt and U. Flechsig, in Gratings and Grating Monochromators for Synchrotron Radiation, Vol. 3150 (SPIE, Bellingham, Washington USA, 1997) pp. 158-170.

[43] O. Chubar, P. Elleaume, S. Kuznetsov, and A. A. Snigirev, Physical optics computer code optimized for synchrotron radiation, in Optical Design and Analysis Software II, Vol. 4769 (International Society for Optics and Photonics, 2002) pp. 145-151.

[44] J. W. Goodman, Introduction to Fourier Optics (Roberts and Company Publishers, Greenwood Village, CO, 2005). 